and whether medication prescription chart was reviewed in $23.9 \%$ case notes.

Intervention We reflected on the results of our audit and discussed the core activities that must be documented, creating standards for our unit. A helpful suggestion from discussion between trainees and nurses led to creation of a 'Ward Round Stamp' that is a simple tick list of 5 issues (see Figure 1) that is used as part of documenting activities on WR.

Strategy for change The results of our observation were discussed in the monthly departmental governance meeting. There were concerns raised about variable standards of ward rounds and their documentation. Few clinicians expressed a view that documenting key aspects of ward rounds are medico-legally important and a thorough documentation supports investigations when case notes are retrospectively scrutinised. Brainstorming further in a focussed team of trainees, consultants and nurses led to the practical decision to use the WR stamp identifying 5 activities that can be ticked. The use of stamp was easy to implement as it was an idea from trainees that their colleagues readily accepted. The use of WR stamp is now routinely discussed at induction whenever the trainees changeover. We are re-auditing the documentation during ward rounds. Interim results show that trainees remember to use the WR stamp on $>80 \%$ occasions. At other times, the general improvement in documentation is noticeable.

Effects of changes There has been changeover of trainees and written feedback suggests that all trainees find paediatric ward rounds to be more structured than other departments they had rotated in. There has been no resistance from trainees or nurses in adapting the WR stamp. We still find occasions when trainees don't use the WR stamp, but these are busier times or locum doctors. Trainees find structured ward rounds are helpful for training future doctors by providing a good example of safe service provision and good documentation.

Lessons learnt I have rotated to another unit and have continued a thorough documentation of all activities during ward rounds.

Message for others I found that lack of structure that can be responsible for poor quality within healthcare and solutions can be simple to devise and implement.

\section{G561(P) SAFE USE OF INSULIN}

${ }^{1,2}{ }^{2}$ Tighe. ${ }^{1}$ Paediatrics, Royal United Hospitals Bath, Bath, UK; ${ }^{2}$ Institute of Child Health, University College London, London, UK

\subsection{6/archdischild-2015-308599.510}

Context The project involves all paediatric inpatients with type 1 diabetes on a general paediatric ward in a district general hospital. The staff involved include paediatric specialist nurses, ward nurses, ward-based doctors, the ward pharmacist and the clinical lead for diabetes.

Problem Errors were occurring with prescribing and administration of insulin, including dosing errors, type of insulin, missed doses and untimely doses. This was leading to poor blood sugar management significant risk to patient safety. Parents were reporting to the diabetes specialist nurses that they lacked confidence in the ward staff's abilities to understand glycaemic control and one teenage patient self-discharged as he felt unsafe.

Assessment of problem and analysis of its causes Construct a process map from a multidisciplinary discussion to diagnose the variations in care during the patient journey. The discussion helped determine:

1. Problems with the prescription chart, e.g. insulin prescribed on different pages; text boxes too small with inappropriate subheadings; not suitable for variable doses.

2. Lack of staff understanding, compounded by high turnover of medical staff sometimes with no paediatric experience and lack of exposure due to low admission rates of diabetics.

3. The lack of clarity of the prescription was leading to an unnecessary step of the nurse calling the doctor to check the quantity of insulin required based on each blood sugar result.

Patient engagement: contact with recent inpatients/their carers to discuss perception of care.

Intervention A search was conducted to see how other departments are approaching this problem and then critically appraise whether these methods would be suitable in our setting. As a starting point we introduced a separate specific insulin prescription chart securely attached to ward drug chart. The chart allowed sufficient space for each type of insulin prescribed, with prompts to guide staff in decision-making regarding dose and to encourage appropriate timing of administration of doses.

Study design Due to the low admission rate of diabetic patients and high risk of harm from drug errors, analysis using real patients would delay the implementation of the safer prescription chart. Therefore initial tests of the chart's suitability were carried out with simulation exercises and repeatedly tested on groups of doctors and nurses, who provided feedback on the charts. The feedback and charts were then analysed by the author along with the ward pharmacist.

Strategy for change The new drug chart will be kept in the same place as the standard drug charts. Use of the chart will be incorporated into the diabetes study day for the nursing staff and the doctors' departmental induction.

Measurement of improvement Run charts of the number of errors found on charts tested out on doctors and nurses with the dummy patients.

Simultaneous analysis of confidence of prescribing and interpretation of prescriptions by nurses.

Once the chart has been through the trust's clinical governance systems and is implemented for real patient use, the incidence of drug errors will be monitored using the incident reporting system and feedback from the ward pharmacist.

Effects of changes It is hoped that there will be a reduction in drug errors and an improvement in the timely, accurate administration of the correct insulin type. It is expected that as a result of improved confidence of the staff and consistency of prescriptions that patient confidence will improve.

Lessons learnt By standardising part of the pathway we predict a reduction in treatment variation.

Message for others Safe insulin prescribing is now mandatory training throughout the NHS. This chart allows this training to be incorporated into best practice.

\section{G562(P) MEDICAL PRODUCTIVITY: QUALITY CARE AND QUALITY TRAINING}

E Coombe, B Lumb, K Luke, C Doherty. Child Health, Cardiff \& Vale University Health Board, Cardiff, UK

\subsection{6/archdischild-2015-308599.511}

Context Inpatient general paediatrics is provided by a variety of medical staff, including Paediatric trainees, General Practice 